\title{
Esophageal dilator as a substitute for the custom stylet to remove the intubating laryngeal airway after tracheal intubation in children
}

\author{
Amit Jain, MD • Kishore Mangal, MD • \\ Atul Jindal, MD
}

Received: 18 December 2010/ Accepted: 21 January 2011/Published online: 5 February 2011

(C) Canadian Anesthesiologists' Society 2011

\section{To the Editor,}

We read with interest the well written Letter to the Editor by Jagannathan et al. reporting use of an airway exchange catheter to facilitate removal of the intubating laryngeal airway (ILA ${ }^{\mathrm{TM}}$ ) after tracheal intubation in children. ${ }^{1}$ The technique prevents accidental extubation of the endotracheal tube (ETT) during the removal of the ILA, with provision for oxygen supplementation throughout the procedure. However, a properly sized pediatric air exchange catheter may not be readily available. We describe use of an esophageal dilator as a substitute for the manufacturer's stabilizing rod when the air- $\mathrm{Q}^{\mathrm{TM}}$ ILA (Mercury Medical, Clearwater, FL, USA) is the conduit for tracheal intubation. An appropriately sized esophageal dilator is equally useful when the Laryngeal Mask Airway (LMA) Classic ${ }^{\mathrm{TM}}$ (LMA North America, San Diego, CA, USA) is used to facilitate tracheal intubation in the pediatric population. The patient's father gave written consent for publication.

An esophageal dilator, also referred to as a bougie tube, is used to dilate the esophagus of a patient in response to medical conditions or treatments that cause esophageal narrowing or tissue shrinkage. Esophageal dilators are a series of flexible dilators of increasing thickness; they comprise a rubber sheath filled with mercury or tungsten and have a tapered tip (Figure, Panel A). We use an 18 Fr

\footnotetext{
A. Jain, MD ( $\square)$

Alchemist Hospitals Ltd., Panchkula, Haryana, India

e-mail: amitvasujain@gmail.com

K. Mangal, MD · A. Jindal, MD

Postgraduate Institute of Medical Education and Research, Chandigarh, India
}

esophageal dilator (Porges Neoplex, France) to replace the custom removal stylet when removing the ILA or LMA after using either as a conduit for tracheal intubation in children (Figure, Panels B-D). Once a successful tracheal intubation through the ILA is achieved, the tapered tip of the bougie can be inserted (to a variable length depending on the size of ETT being used) into the proximal end of the ETT. The clinician can then maintain control of the ETT even when its proximal end is flush with the ILA, thus ensuring that the distal end of the ETT remains in the patient's trachea. The 90-cm long esophageal dilator then allows the ILA to be withdrawn until distal control of the ETT is possible. Further, the ILA lumen is of sufficient size to accommodate the bougie and the pilot balloon of the ETT during withdrawal of the ILA.

The LMA Classic ${ }^{\mathrm{TM}}$ is the conduit used most commonly for tracheal intubation in the pediatric population. ${ }^{1}$ Several techniques have been described in the literature to maintain the control of the ETT while removing the LMA. ${ }^{2}$ However, none provides a perfect solution to the challenge that arises during the removal of the LMA following successful intubation in a patient with a difficult airway, especially when a cuffed ETT is used. We successfully used an 18 Fr esophageal bougie to maintain control of the ETT while removing LMAs in sizes 1.5-3. Though we have never experienced a situation where a size 1 LMA was used as a conduit for tracheal intubation, based on the internal diameter of the size 1 LMA, we speculated that a 16 Fr bougie could be a valuable option to steady the ETT during removal of the LMA. Based on our experience with the use of the LMA Classic ${ }^{\mathrm{TM}}$ as a conduit for tracheal intubation, the proposed specifications for the esophageal bougie as a removal stylet are described in the Table. It should be recognized that the bougie does not provide a lumen for oxygen insufflation during the procedure. 

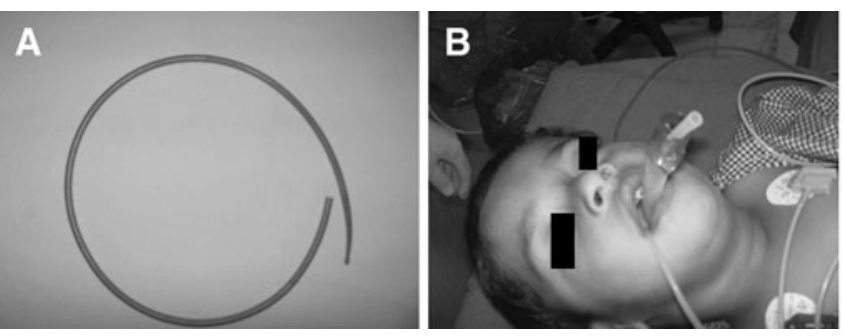

Figure Panel A An esophageal dilator with the tapered distal end. Panels B-D Use of an 18 Fr esophageal bougie to facilitate removal of the Laryngeal Mask Airway (LMA) Classic ${ }^{\mathrm{TM}}$ after successful tracheal intubation through the LMA. Panel B Endotracheal tube (ETT) in position after placement through the LMA. Panel $C$ The
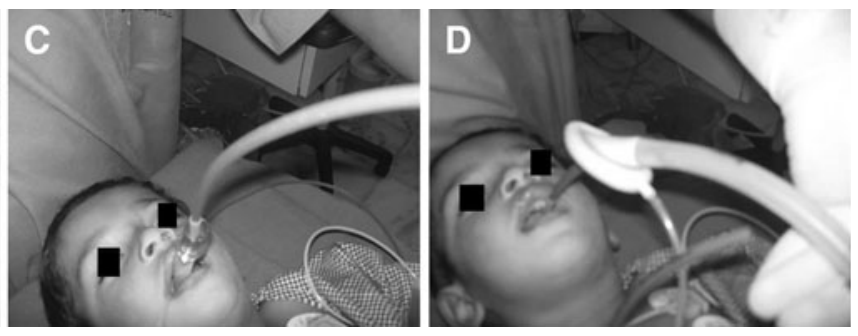

esophageal bougie (distal tapered tip) has been inserted into the distal end of the ETT after the 15-mm ETT adapter has been removed. The ETT remains within the lumen of the LMA. Panel D Removal of the LMA using the esophageal bougie to stabilize the ETT

Table Specifications proposed for using the esophageal bougie as a removal stylet while intubating the trachea through an LMA Classic ${ }^{\mathrm{TM}}$

\begin{tabular}{|c|c|c|c|c|c|}
\hline \multirow[t]{2}{*}{ LMA Classic size } & \multicolumn{2}{|l|}{ LMA Classic } & \multirow{2}{*}{$\begin{array}{l}\text { Largest size ETT (ID, mm) } \\
\text { that can be passed }\end{array}$} & \multirow{2}{*}{$\begin{array}{l}\text { Length at which the } \\
\text { esophageal bougie is } \\
\text { secure in the ETT }\end{array}$} & \multirow{2}{*}{$\begin{array}{l}\text { Esophageal bougi } \\
\text { recommended } \\
\text { (size in Fr) }\end{array}$} \\
\hline & Internal diameter $(\mathrm{mm})$ & Length (mm) & & & \\
\hline 1 & 5.3 & 115 & 3.0 & $23 \mathrm{~mm}$ & 16 \\
\hline 1.0 & 5.3 & 115 & 3.5 & $25 \mathrm{~mm}$ & 16 \\
\hline 1.5 & 6.1 & 135 & 4.0 & $33 \mathrm{~mm}$ & 18 \\
\hline 2 & 7.0 & 155 & 4.5 & $45 \mathrm{~mm}$ & 18 \\
\hline 2.5 & 8.4 & 175 & 5.0 & $50 \mathrm{~mm}$ & 18 \\
\hline 3 & 10 & 220 & 5.5 cuffed & $53 \mathrm{~mm}$ & 18 \\
\hline 3 & 10 & 220 & 6.0 cuffed & $230 \mathrm{~mm}$ & 18 \\
\hline
\end{tabular}

LMA = Laryngeal Mask Airway ${ }^{\text {TM }}$; ETT = endotracheal tube; ID = internal diameter

In conclusion, the $90-\mathrm{cm}$ flexible esophageal bougie with atraumatic tip may provide a useful alternative to the custom stylet for removal of the ILA-LMA after tracheal intubation in children.

Source of funding None.

Conflict of interests None declared.

\section{References}

1. Jagannathan $N$, Kozlowski RJ. An airway exchange catheter facilitates removal of the intubating laryngeal airway after tracheal intubation in children. Can J Anesth 2010; 57: 1044-5.

2. Ellis DS, Potluri PK, O'Flaherty JE, Baum VC. Difficult airway management in the neonate: a simple method of intubating through a laryngeal mask airway. Paediatr Anaesth 1999; 9: 460-2. 